\title{
Structure and electronic properties of transition-metal/Mg bimetallic clusters at realistic temperatures and oxygen partial pressures
}

\author{
Shikha Saini ${ }^{1}$, Debalaya Sarker ${ }^{1}$, Pooja Basera ${ }^{1}$, Sergey V. \\ Levchenko $^{2,3}$, Luca M. Ghiringhelli ${ }^{2}$, Saswata Bhattacharya ${ }^{1 *}$ \\ ${ }^{1}$ Dept. of Physics, Indian Institute of Technology Delhi, New Delhi 110016, India \\ ${ }^{2}$ Theory Department, Fritz-Haber-Institut der Max-Planck-Gesellschaft, Faradayweg 4-6, D-14195 Berlin, Germany \\ ${ }^{3}$ Laboratory of Modelling and Development of New Materials, \\ NUST MISIS, Leninskiy prospekt 4, Moscow 119049, Russia
}

(Dated: April 5, 2018)

\begin{abstract}
Composition, atomic structure, and electronic properties of $\mathrm{TM}_{x} \mathrm{Mg}_{y} \mathrm{O}_{z}$ clusters $(\mathrm{TM}=\mathrm{Cr}, \mathrm{Ni}$, $\mathrm{Fe}, \mathrm{Co}, x+y \leq 3)$ at realistic temperature $T$ and partial oxygen pressure $p_{\mathrm{O}_{2}}$ conditions are explored using the $a b$ initio atomistic thermodynamics approach. The low-energy isomers of the different clusters are identified using a massively parallel cascade genetic algorithm at the hybrid densityfunctional level of theory. On analyzing a large set of data, we find that the fundamental gap $\mathrm{E}_{\mathrm{g}}$ of the thermodynamically stable clusters are strongly affected by the presence of $\mathrm{Mg}$-coordinated $\mathrm{O}_{2}$ moieties. In contrast, the nature of the transition metal does not play a significant role in determining $\mathrm{E}_{\mathrm{g}}$. Using $\mathrm{E}_{\mathrm{g}}$ of a cluster as a descriptor of its redox properties, our finding is against the conventional belief that the transition metal plays the key role in determining the electronic and therefore chemical properties of the clusters. High reactivity may be correlated more strongly with oxygen content in the cluster than with any specific TM type.
\end{abstract}

Keywords: clusters, transition metal, DFT, free energy, reactive environment.

\section{INTRODUCTION}

Transition-metal (TM) nanoparticles/clusters are used as catalysts for a variety of important chemical processes [1 -5]. At nanoscale, the particles acquire unique properties not found in the bulk TMs. The size of the nanoparticles is an additional parameter that affects their electronic and chemical properties and can be used to control the activity and selectivity of the catalyst. Moreover, the particles containing two or more transition or other metals can be synthesized, which tremendously increases the possibilities for tuning their functional properties. Together with these possibilities, new challenges arise for the design of more efficient and stable nanocatalysts. In particular, due to the increased complexity, the atomic structure and stability of the nanoparticles is harder to determine. Moreover, the particles usually operate in a reactive atmosphere, containing, e.g., oxygen. The interaction of the particles with oxygen will eventually result in their partial or complete oxidation, whether it is intended or not [6]. In fact, in some applications [7], the particles are created by a reduction of the oxide in hydrogen. Thus, both oxidized and reduced metal atoms are usually present at the surface, with their relative concentrations depending on the temperature and oxygen partial pressure. Disentangling the role of different oxidation states in a catalytic process is extremely challenging.

In view of the above, it is important to provide the-

\footnotetext{
*saswata@physics.iitd.ac.in
}

oretical guidance to experiment and technology on the composition and atomic structure of metal particles in a reactive atmosphere, in particular in the ubiquitous presence of oxygen. Stability being a key element for the desired functioning of a catalyst, it is a prerequisite to know the most stable phase of a particle/cluster in the reactive atmosphere. There are both experimental and theoretical reports stating the existence of particular phases viz. $\mathrm{Ni}_{4} \mathrm{O}_{5}$ clusters (on top of $\mathrm{MgO}$ substrate) under oxygen atmosphere [8]. However, no quantitative information has been provided so far to understand which structures/compositions are stable at what experimental conditions.

We report here an $a b$ initio atomistic thermodynamics 9] study of TM clusters supported on $\mathrm{MgO}$, as well as unsupported TM-Mg clusters in an oxygen atmosphere. Gas-phase clusters are often used as model systems to study structural and compositional variability of the clusters at realistic conditions both experimentally and theoretically. Although such models cannot capture the full complexity of the supported nano-catalysts, they provide the necessary knowledge to disentangle various effects determining the catalytic activity. Using a massively parallel $a b$ initio cascade genetic algorithm [6], we identify stable and metastable structures of the clusters at a hybrid density functional theory (DFT) level, in order to overcome the difficulties of the standard Local Density Approximation (LDA) and Generalized Gradient Approximation (GGA) exchange-correlation functionals in describing localization of $d$-electrons in TMs, as well as the charge transfer from metal atoms to oxygen [10]. We present an exhaustive and reliable database of small ternary $\mathrm{TM}_{x} \mathrm{Mg}_{y} \mathrm{O}_{z}$ clusters $(\mathrm{TM}=\mathrm{Cr}, \mathrm{Ni}, \mathrm{Fe}, \mathrm{Co} ; x+y$ 
$\leq 3, z=1,2, \ldots, 12)$. Among a variety of TMs, Cr, Ni, $\mathrm{Fe}$, and $\mathrm{Co}$ are of special interest to the catalytic research community because of their wide applications viz. in partial oxidation of $\mathrm{CH}_{4}$ 7, $11-15$, selective oxidation of alkanes [16 19], alcohols 20, 21], olefins [22], and aromatics [23], selective reduction of nitrogen oxides [24], and oxidation of hydrogen sulfide [25]. $\mathrm{MgO}$ is often used as a support in these applications, or as a component of an oxide solid solution catalyst [7]. The high thermal stability and low cost are two important factors that make $\mathrm{MgO}$ to be one of the best choices for catalytic applications [8, 26].

A simple descriptor that captures both the nature of the reactive oxygen centers and the tendency of adjacent TM centers to gain electron density is the ligand-to-metal charge transfer excitation energy [27]. For oxides having TM centres in their highest oxidation states, this ligandto-metal charge transfer excitation energy typically corresponds to the band-gap energy. For a cluster, electron transfer to/from it is more appropriately described by the electron affinity (EA) and ionization potential (IP), respectively. Therefore, the clusters having a high EA and a low IP are the ones having a low fundamental gap $\left(E_{\mathrm{g}}=\right.$ EA - IP). Clusters possessing a high EA and/or a low IP may be expected to be better catalysts because they would accept or donate an electron more readily [28, 29]. Thus, fundamental gap $\mathrm{E}_{\mathrm{g}}$ can be considered as an important feature of the clusters that determines their redox properties, and in many cases can serve as a descriptor of the catalytic activity [27, 30 36].

We therefore analyze the dependence of $\mathrm{E}_{\mathrm{g}}$ of the clusters on composition, temperature, and $\mathrm{O}_{2}$ pressure, and determine the explicit role of different atomic species to modulate the $\mathrm{E}_{\mathrm{g}}$ values. Strikingly, we find that the nature of TM plays a negligible role in tuning the $\mathrm{E}_{\mathrm{g}}$ in $\mathrm{TM}_{x} \mathrm{Mg}_{y} \mathrm{O}_{z}$ clusters. Rather, $\mathrm{E}_{\mathrm{g}}$ is strongly correlated with the presence of $\mathrm{Mg}$-coordinated $\mathrm{O}_{2}$ (i.e., the oxygen atoms directly attached with $\mathrm{Mg}$ atom) and is found to be minimum under O-rich conditions (i.e., $\mathrm{z}>\mathrm{x}+\mathrm{y}$, when $T \approx 200 \mathrm{~K}-450 \mathrm{~K}$ and $\left.p_{\mathrm{O}_{2}} \approx 10^{-6}-10^{10} \mathrm{~atm}\right)$. The analysis of our high-throughput data suggests that the tunability of the cluster chemical properties may be limited at given $\left(T, p_{\mathrm{O}_{2}}\right)$ conditions, but is strongly dependent on the conditions.

\section{METHODOLOGY}

We have considered first a wide range of $\mathrm{TM}_{x} \mathrm{Mg}_{y} \mathrm{O}_{z}$ cluster compositions, where $z$ is determined by thermal equilibrium with the environment at given temperature $T$ and partial oxygen pressure $p_{\mathrm{O}_{2}}$ [48]. In order to get the minimum energy configurations, for each stoichiometry, the total energy is minimized with respect to both geometry and spin state. The low-energy structures (including the global minimum) are generated from an ex- haustive scanning of the potential energy surface (PES) using our recent implementation of cascade genetic algorithm (GA) [6, 10]. The term "cascade" means a multi-stepped algorithm where successive steps employ higher level of theory and each of the next level takes information obtained at its immediate lower level. Typically here the cascade GA starts from a DFT with semilocal xc-functionals and goes up to DFT with hybrid xc-functionals. This GA algorithm's implementation is thoroughly benchmarked and it's efficiency is validated (w.r.t more advanced theory) in detail in Ref. [6].

We have performed the DFT calculations using FHIaims, which is an all electron code with numerical atom centred basis sets [37]. The low-energy GA structures are further optimized at a higher level settings, where energy minimization is performed with vdW-corrected PBE $(\mathrm{PBE}+\mathrm{vdW})$ functional, "tight - tier 2" settings, and force tolerance was set to better than $10^{-5} \mathrm{eV} / \AA$. The van der Waals correction is calculated as implemented in Tkatchenko-Scheffler scheme [38]. The total singlepoint energy is calculated afterwards on top of this optimized structure via vdW-corrected-PBE0 [39] hybrid xcfunctional $(\mathrm{PBE} 0+\mathrm{vdW})$, with "tight - tier 2" settings [49]. Note that PBE+vdW strongly overestimates the stability of oxide-clusters under O-rich conditions (i.e. with larger $z$ values) as reported for the case of $\mathrm{Mg}_{y} \mathrm{O}_{z}$ clusters [10]. This gives a qualitatively wrong prediction that adsorption of $\mathrm{O}_{2}$ could be favored over desorption up to a large excess of oxygen. Such behavior is not confirmed by hybrid functionals [e.g. HSE06, PBE0] employed in our calculations. For the data set we have used, the difference in energetics of PBE0 and HSE06 is always within $0.04 \mathrm{eV}$. The spin states of the clusters are also sometimes different as found by PBE and PBE0/HSE06. Thus, all our results are thoroughly tested and benchmarked w.r.t hybrid xc-functionals (PBE0) using "tight" numerical settings and tier 2 basis set. For estimation of (vertical) electron affinity (VEA), (vertical) ionization potential (VIP), and fundamental gap $\left(\mathrm{E}_{\mathrm{g}}=\mathrm{VEA}\right.$ - VIP) we have used the $G_{0} W_{0} @ \mathrm{PBE} 0$ approach with "reallytight" numerical settings and tier 4 basis set [37].

The free energy of the isomers [50] is then calculated as a function of $T$ and $p_{\mathrm{O}_{2}}$ for each stoichiometry using the $a b$ initio atomistic thermodynamics (aiAT) approach. The concept of aiAT was successfully applied initially for bulk semiconductors [40, 41], and later applied to the study of oxide formation at the surface of some TMs and other materials [42 45]. We have recently extended this approach to clusters in a reactive atmosphere [6, 10, 46, 47]. From different cluster compositions and structures with the lowest free energy the thermodynamic phase diagram can be constructed as a function of $T$ and $p_{\mathrm{O}_{2}}$. Such phase diagrams are shown in Fig. 2 At a given $T$, $p_{\mathrm{O}_{2}}$, and $x, y$, the stable stoichiometry of a $\mathrm{TM}_{x} \mathrm{Mg}_{y} \mathrm{O}_{z}$ cluster is determined via aiAT, i.e., by minimizing the 
Gibbs free energy of formation $\Delta G_{f}\left(T, p_{\mathrm{O}_{2}}\right)$.

$$
\begin{array}{r}
\Delta G_{f}\left(T, p_{\mathrm{O}_{2}}\right)=F_{\mathrm{TM}_{x} \mathrm{Mg}_{y} \mathrm{O}_{z}}(T)-F_{\mathrm{TM}_{x} \mathrm{Mg}_{y}}(T) \\
-z \times \mu_{\mathrm{O}}\left(T, p_{\mathrm{O}_{2}}\right)
\end{array}
$$

Here, $F_{\mathrm{TM}_{x} \mathrm{Mg}_{y} \mathrm{O}_{z}}(T)$ and $F_{\mathrm{TM}_{x} \mathrm{Mg}_{y}}(T)$ are the Helmholtz free energies of the $\mathrm{TM}_{x} \mathrm{Mg}_{y} \mathrm{O}_{z}$ and the pristine $\mathrm{TM}_{x} \mathrm{Mg}_{y}$ cluster [51] and $\mu_{\mathrm{O}}\left(T, p_{\mathrm{O}_{2}}\right)$ is the chemical potential of oxygen. As explained in Ref. [6], $F_{\mathrm{TM}_{x} \mathrm{Mg}_{y} \mathrm{O}_{z}}(T)$ and $F_{\mathrm{TM}_{x} \mathrm{Mg}_{y}}(T)$ are calculated as the sum of DFT total energy, DFT vibrational free energy (up to harmonic approximation), translational, rotational, symmetry and spin-degeneracy free-energy contributions. The dependence of $\mu_{\mathrm{O}}\left(T, p_{\mathrm{O}_{2}}\right)$ on $T$ and $p_{\mathrm{O}_{2}}$ is calculated using the ideal (diatomic) gas approximation with the same DFT functional as for the clusters. The phase diagram for a particular $\mathrm{TM}_{x} \mathrm{Mg}_{y} \mathrm{O}_{z}$ is constructed by identifying the lowest free-energy structures at each $T, p_{\mathrm{O}_{2}}$.

\section{RESULTS AND DISCUSSIONS}

In order to understand whether the presence of $\mathrm{MgO}$ substrate is crucial or not in the stability analysis of TM clusters, we have considered two cases: (a) stand-alone $\mathrm{Ni}_{4}$ cluster in an oxygen environment, and (b) $\mathrm{Ni}_{4}$ cluster on $\mathrm{MgO}$ substrate in oxygen environment. It is indeed evident from Fig. 1 that ignoring the substrate's effect in stability analysis of the TM clusters is not meaningful as the substrate (here $\mathrm{MgO}$ ) plays a notable role to the stability of the TM clusters under oxygen atmosphere. The corresponding $T-p_{\mathrm{O}_{2}}$ phase diagrams are plotted in Fig. 1 by minimizing the Gibbs' free energy of formation of the all possible configurations (see methodology for details) [52]. Note that in Ref. [8] the experimental evidence of $\mathrm{Ni}_{4} \mathrm{O}_{5}$ phase is observed from STM analysis. This phase is stable at a given experimental condition in the phase diagram only when we have considered the $\mathrm{MgO}$ substrate, while the concerned phase is totally missing when substrate effect is not included in our calculations. This clearly suggests that even if consideration of the support is computationally demanding, it is still a necessary condition to understand the stability of the TM clusters correctly.

In order to explore the explicit effect of the support for a wide range of compositions and configurations, here we model the $\mathrm{TM}-\mathrm{MgO}$ system by means of small bi-metallic $\mathrm{TM}_{x} \mathrm{Mg}_{y} \mathrm{O}_{z}$ clusters. An exhaustive set of $\mathrm{TM}_{x} \mathrm{Mg}_{y} \mathrm{O}_{z}$ clusters are generated including all the possible structural and composition motifs, oxidations states, electronic spin, symmetry etc. as if when $\mathrm{MgO}$-supported TM clusters are prepared from a solid solution. Following this thermodynamic phase diagrams are calculated for all possible combinations of $x+y \leq 3(y \neq 0)$ in $\mathrm{TM}_{x} \mathrm{Mg}_{y} \mathrm{O}_{z}$ clusters, and the most stable compositions and configu-

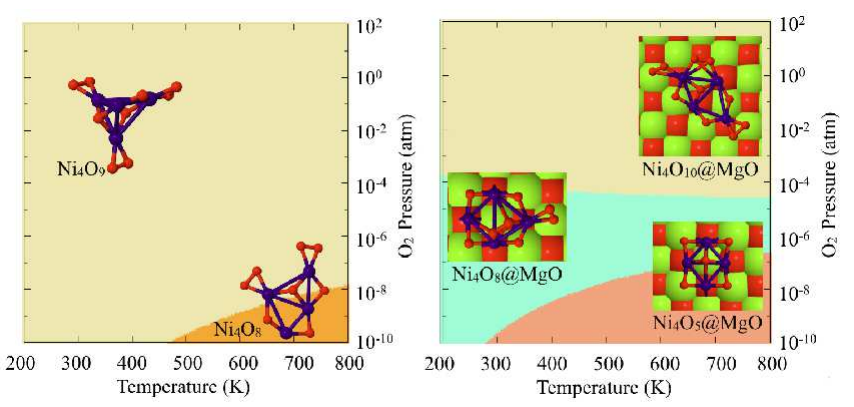

FIG. 1: $T-p_{\mathrm{O}_{2}}$ phase diagram (see text) of $\mathrm{Ni}_{4}$ clusters under oxygen atmosphere with (right) and without (left) $\mathrm{MgO}$ substrate. The red, navyblue, and green color-balls represent $\mathrm{O}$, $\mathrm{Ni}$ and $\mathrm{Mg}$ respectively.

rations are identified. Prior to this, all the global minimum structures of the clusters are determined by cascade GA. In Fig. 2(a-d) we show phase diagrams of a set of $\mathrm{TM}_{1} \mathrm{Mg}_{2} \mathrm{O}_{z}$ clusters, where four different TMs viz. Cr, $\mathrm{Co}, \mathrm{Ni}, \mathrm{Fe}$ are investigated respectively. The most stable configurations and the respective stable phases are shown within a window of experimentally achievable environmental conditions $\left(T, p_{\mathrm{O}_{2}}\right.$ window). From Fig. 2 $\mathrm{a}$, we see for $\mathrm{TM}=\mathrm{Cr}$ and $x=1, y=2$ at low $T$ and high $p_{\mathrm{O}_{2}}, \mathrm{Cr}_{1} \mathrm{Mg}_{2} \mathrm{O}_{8}$ is the most stable phase, while at high $T$ and low $p_{\mathrm{O}_{2}} \mathrm{Cr}_{1} \mathrm{Mg}_{2} \mathrm{O}_{5}$ is the most stable phase.

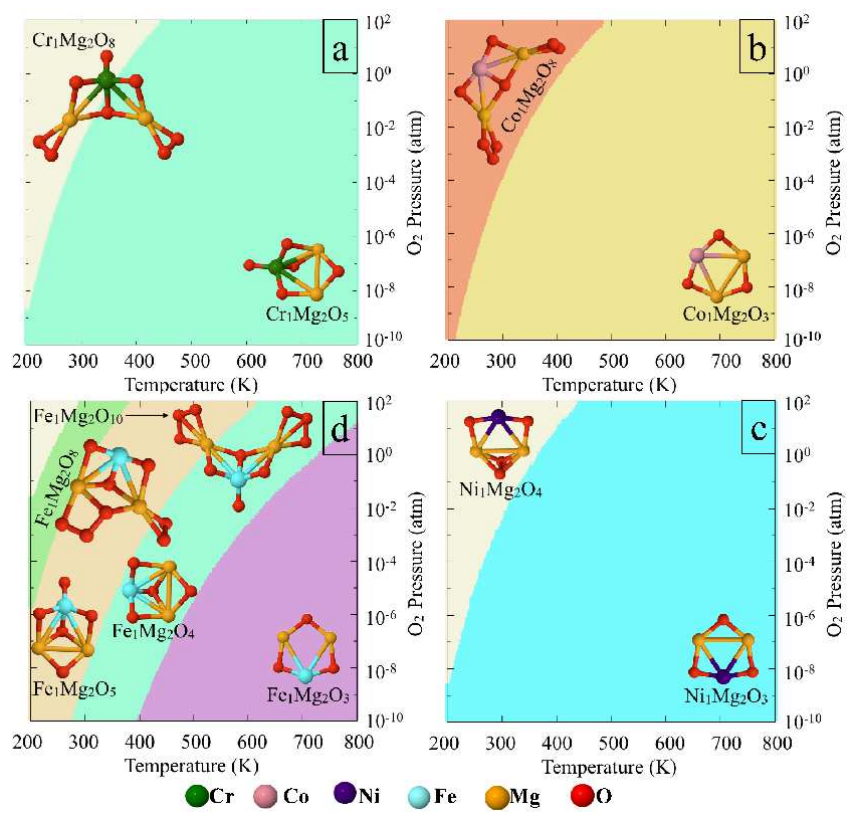

FIG. 2: (color online) The most stable $\mathrm{TM}_{x} \mathrm{Mg}_{y} \mathrm{O}_{z}$ clusters at various temperatures and pressures under thermodynamic equilibrium. In $\mathrm{TM}_{x} \mathrm{Mg}_{y} \mathrm{O}_{z}$ clusters, $\mathrm{TM}=\mathrm{Cr}$ (a), $\mathrm{Co}(\mathrm{b}), \mathrm{Ni}$ (c) and $\mathrm{Fe}(\mathrm{d})$. The geometries are optimized with $\mathrm{PBE}+\mathrm{vdW}$, and the electronic energy is calculated using HSE06+vdW. The vibrational free energy is computed under harmonic approximations. 

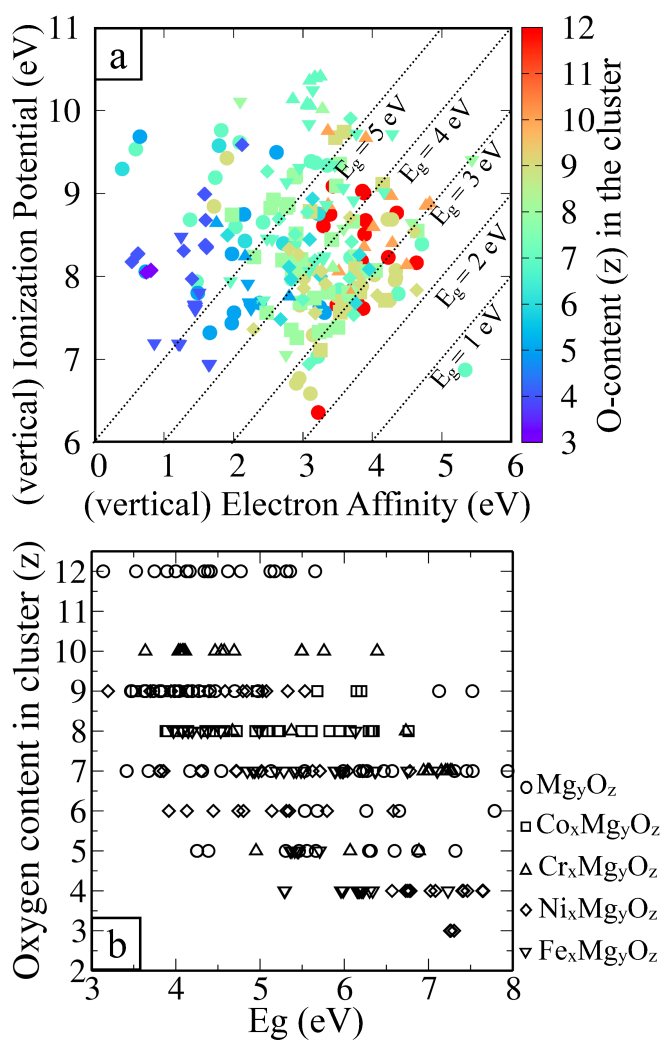

FIG. 3: (color online) (a) VIP vs VEA for the low energy isomers stable at an experimentally achievable environmental condition for all different cluster sizes $(x+y \leq 3)$. The symbols represent the nature of the TM atoms in the clusters, while the colour is set as per the amount of oxygen content (as shown by the colour bar) in the cluster. The loci of constant $\mathrm{E}_{\mathrm{g}}$ are indicated by diagonal lines. (b) $\mathrm{E}_{\mathrm{g}}$ vs oxygen content $(z)$ is plotted for all the stable isomers at realistic conditions (see text for details).

For $\mathrm{TM}=\mathrm{Co}$ and $\mathrm{Ni}$ (see Fig. 2b and Fig. 2r) these are respectively $\left(\mathrm{Co}_{1} \mathrm{Mg}_{2} \mathrm{O}_{8}, \mathrm{Co}_{1} \mathrm{Mg}_{2} \mathrm{O}_{3}\right),\left(\mathrm{Ni}_{1} \mathrm{Mg}_{2} \mathrm{O}_{4}\right.$, $\mathrm{Ni}_{1} \mathrm{Mg}_{2} \mathrm{O}_{3}$ ). However for $\mathrm{TM}=\mathrm{Fe}$ (see Fig. 2 $\mathrm{d}$ ) at low $T$ and high $p_{\mathrm{O}_{2}}$ there are a lot of competing isomers viz. $\mathrm{Fe}_{1} \mathrm{Mg}_{2} \mathrm{O}_{10}, \mathrm{Fe}_{1} \mathrm{Mg}_{2} \mathrm{O}_{8}$ and $\mathrm{Fe}_{1} \mathrm{Mg}_{2} \mathrm{O}_{5}$. At a moderate $T$ and moderate $p_{\mathrm{O}_{2}}$ i.e. $T \approx 300 \mathrm{~K}, p_{\mathrm{O}_{2}} \approx 10^{-6} \mathrm{~atm}$ there is competition between two phases viz. $\mathrm{Fe}_{1} \mathrm{Mg}_{2} \mathrm{O}_{4}$ and $\mathrm{Fe}_{1} \mathrm{Mg}_{2} \mathrm{O}_{5}$. At high $T$ and low $p_{\mathrm{O}_{2}}$ the most stable phase is $\mathrm{Fe}_{1} \mathrm{Mg}_{2} \mathrm{O}_{3}$. Thus, we observe a general trend that usually O-rich clusters are more stable at low $T$ and moderate to high $p_{\mathrm{O}_{2}}$ (i.e. upto $\approx 10^{10} \mathrm{~atm}$ ).

The electronic structure of the clusters determines their reactivity, and can serve as a descriptor of the catalytic activity. Under reaction conditions, the catalyst comprises of a wide range of structures including different number of atoms with various oxidation states, all of which could be active to some extent in the catalytic reaction. Therefore, after identifying the most stable compositions (i.e., specific $x, y$, and $z$ in stable phases of $\mathrm{TM}_{x} \mathrm{Mg}_{y} \mathrm{O}_{z}$ ), we have studied electronic structure of not only the global minimum isomer of that given composition but also all the low-energy isomers lying within an energy window of $0.5 \mathrm{eV}$ from the global minimum 53 .

In Fig. 3a we show VEA and VIP values (at the level of $\left.G_{0} W_{0} @ \mathrm{PBE} 0\right)$ of all such thermodynamically stable isomers. Typically we have included data for $\mathrm{TM}_{1} \mathrm{Mg}_{1} \mathrm{O}_{z}$, $\mathrm{TM}_{1} \mathrm{Mg}_{2} \mathrm{O}_{z}, \mathrm{TM}_{2} \mathrm{Mg}_{1} \mathrm{O}_{z}$ with $\mathrm{TM}=\mathrm{Cr}, \mathrm{Co}, \mathrm{Ni}, \mathrm{Fe} . \mathrm{We}$ have also included data for clusters without TM atoms, i.e., $\mathrm{Mg}_{y} \mathrm{O}_{z}(y=1-3)$ clusters. The symbols are selected as per the type of the TM atoms in the clusters, while the colour of the symbols are selected based on the amount of oxygen content in the cluster. The diagonal lines are drawn to represent the corresponding constant $\mathrm{E}_{\mathrm{g}}$, which is evaluated as the absolute difference between VIP and VEA. In Fig. $3 \mathrm{~b}$ the $\mathrm{E}_{\mathrm{g}}$ for the clusters are widely scattered at all different sizes and nature of TM atoms. In Fig. 3b we have explicitly shown $\mathrm{E}_{\mathrm{g}}$ with different no. of oxygen content $(z)$ in the clusters. Clearly, it is not following any trends except the $\mathrm{E}_{\mathrm{g}}$ for O-deficient clusters (i.e. $z \leq x+y$ ) are consistently in the higher side (see, bluish points in the Fig. [3a and the region around low oxygen content $(z \approx 3-5))$. Thus for sure it can be concluded from Fig. 3 that O-deficient clusters (mostly stable at high $T$ and low $p_{\mathrm{O}_{2}}$ ) are not good for catalysis. However, for O-rich clusters (i.e. $z \geq x+y$ ) both high and low $\mathrm{E}_{\mathrm{g}}$ are found (see Fig. $3 \mathrm{~b}$ around $z \geq 6$ ). This observation hints towards the fact that some structural feature in these O-rich clusters might act as active site that dominates the low $\mathrm{E}_{\mathrm{g}}$ value. Moreover, the invariance of the $\mathrm{E}_{\mathrm{g}}$ values for different TM clusters with same O-content emphasizes that it is not the nature of TM atoms but probably some active centers originating from oxygen moieties that affect the fundamental gap. Note that it is common understanding that the former (i.e., nature of the TM atoms) is solely responsible for making the material a good catalyst $7,11,14,14,15,15]$.

We have next divided our clusters into two sets viz. (i) having low gap $(\leq 4 \mathrm{eV})$ and (ii) having high gap (> $4 \mathrm{eV}$ ). Following this, the vibrational frequencies (corresponding O-vibrations) of all the structures are care-

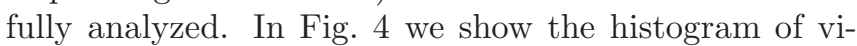
brational frequencies by including an average of all vibrational spectra ranging $1000-1200 \mathrm{~cm}^{-1}$. Clearly the peak corresponding to the frequency $1151 \mathrm{~cm}^{-1}$ of the $\mathrm{O}_{2}$ moiety bonded to the $\mathrm{Mg}$ atom, is missing for all clusters with the $\mathrm{E}_{\mathrm{g}}>4 \mathrm{eV}$ (see in Fig. 4). This means clusters with low $\mathrm{E}_{\mathrm{g}}$ have some correlation with this $\mathrm{Mg}$ coordinated $\mathrm{O}_{2}$ moieties, while clusters with higher $\mathrm{E}_{\mathrm{g}}$ do not have this structural feature.

To unravel the particular role of these $\mathrm{O}_{2}$ moieties in the $\mathrm{E}_{\mathrm{g}}$, we examined the density of states (DOS) pro-

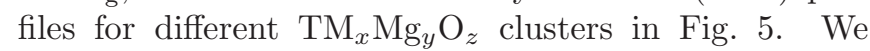
can see the HOMO-LUMO gap in $\mathrm{Cr}_{1} \mathrm{Mg}_{2} \mathrm{O}_{8}$ is smaller than in $\mathrm{Cr}_{1} \mathrm{Mg}_{2} \mathrm{O}_{5}$. Presence of spin-polarised states near fermi-energy $\left(\mathrm{E}_{f}\right)$ leads to the reduced HOMOLUMO gap in the oxygen-rich phase. Further, compar- 


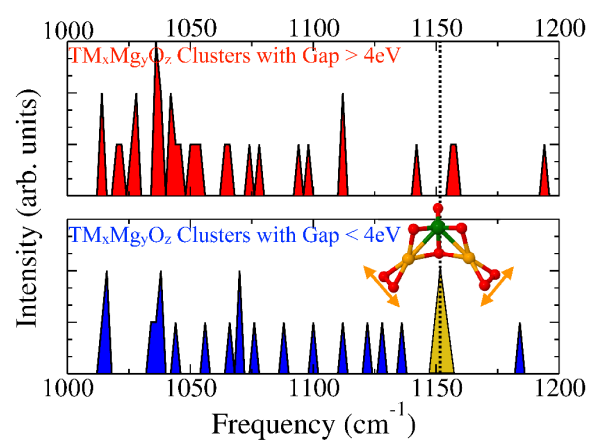

FIG. 4: (color online) Histogram of vibrational frequencies ranging from 1000-1200 $\mathrm{cm}^{-1}$ for clusters (i) gap $>4 \mathrm{eV}$ (top panel) (ii) gap $\leq 4 \mathrm{eV}$ (bottom panel).

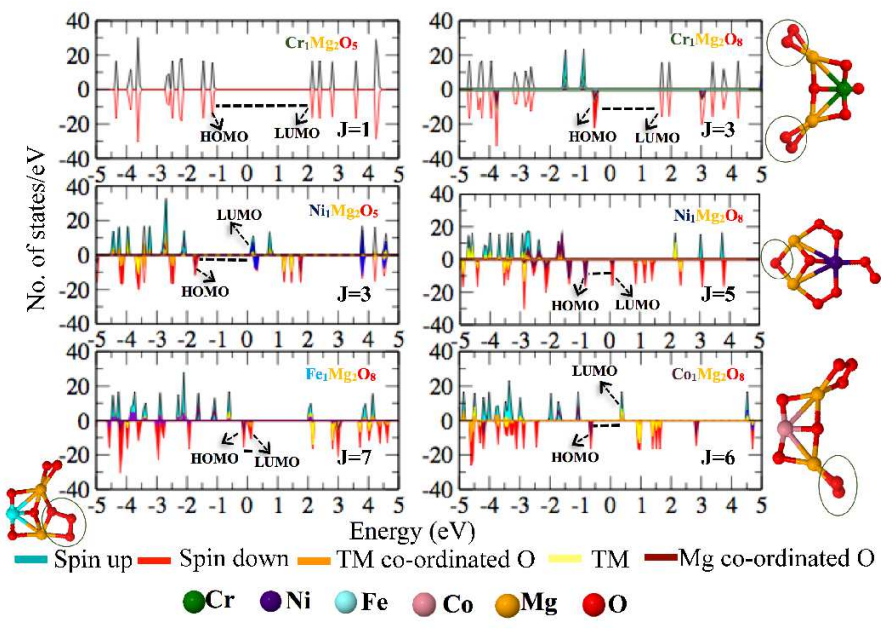

FIG. 5: (color online) Atom-projected spin polarised density of states of different $\mathrm{TM}_{x} \mathrm{Mg}_{y} \mathrm{O}_{z}$ clusters. The corresponding spin multiplicity $(\mathrm{J})$ is also given in the respective plots.

ing top panel in Fig. 5 we observe that the presence of TM (Cr) atom has minimal effect on the spin polarized states near $\mathrm{E}_{f}$. These states occur only due to the extra oxygen atoms in $\mathrm{Cr}_{1} \mathrm{Mg}_{2} \mathrm{O}_{8}$, whereas they are absent in case of $\mathrm{Cr}_{1} \mathrm{Mg}_{2} \mathrm{O}_{5}$. To further understand if our observation is coincidental for $\mathrm{Cr}$ clusters or not, we have next plotted the same for $\mathrm{Ni}$ clusters in Fig. 5 (middle panel). We see here again that the HOMO-LUMO gap is smaller for the oxygen-rich phase, i.e., $\mathrm{Ni}_{1} \mathrm{Mg}_{2} \mathrm{O}_{8}$ in comparison to $\mathrm{Ni}_{1} \mathrm{Mg}_{2} \mathrm{O}_{5}$. By having the insight from the atom-projected DOS, we conclude that the spin-polarized states are indeed the states of $\mathrm{Mg}$-coordinated oxygen atoms in the O-rich clusters, which have caused a reduced $\mathrm{E}_{\mathrm{g}}$ in these clusters. Next in Fig. 5 (bottom panel), we see the O-rich phases of $\mathrm{Fe}$ and Co clusters respectively, which are having smaller $\mathrm{E}_{\mathrm{g}}$ in comparison to their Oreduced phases. Again the atom-projected DOS indicate that the $\mathrm{Mg}$ coordinated $\mathrm{O}$-atoms are playing pivotal role in the states near $\mathrm{E}_{f}$ and hence are responsible for the reduced $\mathrm{E}_{\mathrm{g}}$ in these clusters. The $\mathrm{Fe}_{1} \mathrm{Mg}_{2} \mathrm{O}_{8}$ cluster has the lowest $\mathrm{E}_{\mathrm{g}}$ among all because of the presence of spinpolarized $3 d$ states of $\mathrm{Fe}$ near $\mathrm{E}_{f}$. However, it can easily be seen from the comparison of $z=5$ and $z=8$ clusters that it is not the nature of TM atoms, but the states of $\mathrm{Mg}$ coordinated $\mathrm{O}$ atoms in the O-rich phases of each $\mathrm{TM}_{x} \mathrm{Mg}_{y} \mathrm{O}_{z}$ cluster that play the crucial role in controlling the $\mathrm{E}_{\mathrm{g}}$. Irrespective to any specific TM atoms, TM has always states at the LUMO level, whereas the HOMO keeps on shifting depending on oxygen content in the cluster. Under O-rich condition, the clusters usually have $\mathrm{O}_{2}$ (containing loosely bound electrons) coordinated with $\mathrm{Mg}$ atom. This gives rise to occupied states near $\mathrm{E}_{f}$ yielding a smaller $\mathrm{E}_{\mathrm{g}}$.

\section{CONCLUSION}

In summary, we have used a robust first-principles approach to understand the stability and electronic structure of ternary $\mathrm{TM}_{x} \mathrm{Mg}_{y} \mathrm{O}_{z}$ clusters at realistic temperatures and oxygen partial pressures. The low-energy isomers of different composition are determined by cascade genetic algorithm. Following that, the stable compositions are found by minimizing Gibbs free energy of formation using aiAT methodology (within harmonic approximation for the vibrational contributions). From the analysis of the electronic structure of the clusters, we establish that the presence of $\mathrm{Mg}$-coordinated-O in $\mathrm{O}$ rich clusters (i.e. $z>x+y$ ) correlates with low $\mathrm{E}_{\mathrm{g}}$, whereas the nature of TM atoms is insignificant. We further note that these $\mathrm{Mg}$ coordinated $\mathrm{O}_{2}$ moieties have unpaired electrons that give rise to multiple degenerate spin states. The latter play key role in producing a reduced $\mathrm{E}_{\mathrm{g}}$ from the $\mathrm{O}_{2}$ moieties at an environmental condition $T \approx 200 \mathrm{~K}$ - $450 \mathrm{~K}$ and $p_{\mathrm{O}_{2}} \approx 10^{-6}-10^{10}$ atm; while at higher temperature and lower pressure stable clusters have less O-content. These observations lead us to argue that the chemical (redox) properties of mixed metal oxide clusters are sensitive to the oxygen content controlled by $T$ and $p_{\mathrm{O}_{2}}$ rather than to the specific transition metal type. We hope that this fining will be helpful for understanding experiments with gas-phase and supported metal clusters at realistic $\left(T, p_{\mathrm{O}_{2}}\right)$ conditions, and for designing new functional nano-materials.

\section{ACKNOWLEDGEMENT}

SS acknowledges CSIR, India, for the senior research fellowship [grant no. 09/086(1231)2015-EMR-I]. PB acknowledges UGC, India, for the junior research fellowship [grant no. 20/12/2015(ii)EU-V]. SB and DS acknowledge the financial support from YSS-SERB research grant, DST, India (grant no. YSS/2015/001209). SB thanks Bryan R. Goldsmith for critically reading the manuscript. 
SVL is grateful for his support from the German Science Foundation in the frame of the Berlin Cluster of Excellence "Unifying Concepts in Catalysis", and to the Ministry of Education and Science of the Russian Federation in the framework of Increase Competitiveness Program of NUST MISIS (No K2-2016-013) implemented by a governmental decree dated 16 March 2013, No 211. We acknowledge the High Performance Computing (HPC) facility at IIT Delhi for computational resources. We thank Karsten Reuter for many helpful discussions.

[1] E. W. McFarland and H. Metiu, Chemical reviews 113, 4391 (2013).

[2] K. Zemski, D. Justes, and A. Castleman, Studies of metal oxide clusters: Elucidating reactive sites responsible for the activity of transition metal oxide catalysts (2002).

[3] J. Guzman and B. C. Gates, Dalton Transactions pp. 3303-3318 (2003).

[4] L. Liu, Z. Ji, W. Zou, X. Gu, Y. Deng, F. Gao, C. Tang, and L. Dong, Acs Catalysis 3, 2052 (2013).

[5] G. Zhao, F. Yang, Z. Chen, Q. Liu, Y. Ji, Y. Zhang, Z. Niu, J. Mao, X. Bao, P. Hu, et al., Nature communications 8, 14039 (2017).

[6] S. Bhattacharya, S. V. Levchenko, L. M. Ghiringhelli, and M. Scheffler, New Journal of Physics 16, 123016 (2014).

[7] Y. H. Hu and E. Ruckenstein, Catalysis Reviews 44, 423 (2002).

[8] M. Smerieri, J. Pal, L. Savio, L. Vattuone, R. Ferrando, S. Tosoni, L. Giordano, G. Pacchioni, and M. Rocca, The Journal of Physical Chemistry Letters 6, 3104 (2015), pMID: 26267209, http://dx.doi.org/10.1021/acs.jpclett.5b01362, URL http://dx.doi.org/10.1021/acs.jpclett.5b01362

[9] M. Scheffler and C. Weinert, in Defects in Semiconductors, edited by H. J. v. Bardeleben (Trans. Tech. Publ. Ltd, Switzerland, 1986), pp. 25-30.

[10] S. Bhattacharya, S. V. Levchenko, L. M. Ghiringhelli, and M. Scheffler, Physical review letters 111, 135501 (2013).

[11] R. Subbaraman, D. Tripkovic, K.-C. Chang, D. Strmcnik, A. P. Paulikas, P. Hirunsit, M. Chan, J. Greeley, V. Stamenkovic, and N. M. Markovic, Nature materials 11, 550 (2012).

[12] M. K. Bates, Q. Jia, H. Doan, W. Liang, and S. Mukerjee, ACS Catalysis 6, 155 (2015).

[13] C. Liu, T. R. Cundari, and A. K. Wilson, The Journal of Physical Chemistry C 116, 5681 (2012).

[14] J. D. Aiken and R. G. Finke, Journal of Molecular Catalysis A: Chemical 145, 1 (1999).

[15] C. L. Hill and C. M. Prosser-McCartha, Coordination Chemistry Reviews 143, 407 (1995).

[16] S. Albonetti, F. Cavani, and F. Trifiro, Catalysis Reviews 38, 413 (1996).

[17] S. Albonetti, F. Cavani, F. Trifiro, P. Venturoli, G. Calestani, M. L. Granados, and J. G. Fierro, Journal of catalysis 160, 52 (1996).

[18] M. A. Bañares, Catalysis Today 51, 319 (1999).

[19] B. Hodnett, Catalysis Reviews Science and Engineering
27, 373 (1985).

[20] W. C. Vining, A. Goodrow, J. Strunk, and A. T. Bell, Journal of Catalysis 270, 163 (2010).

[21] W. Zhang, A. Desikan, and S. T. Oyama, The Journal of Physical Chemistry 99, 14468 (1995).

[22] R. K. Grasselli, Topics in Catalysis 15, 93 (2001).

[23] V. Nikolov, D. Klissurski, and A. Anastasov, Catalysis Reviews 33, 319 (1991).

[24] R. M. Heck, Catalysis Today 53, 519 (1999).

[25] K.-T. Li, M.-Y. Huang, and W.-D. Cheng, Industrial \& engineering chemistry research 35, 621 (1996).

[26] J. Pal, M. Smerieri, E. Celasco, L. Savio, L. Vattuone, and M. Rocca, Phys. Rev. Lett. 112, 126102 (2014), URL https://link.aps.org/doi/10.1103/PhysRevLett.112.126102

[27] A. ?. Getsoian, Z. Zhai, and A. T. Bell, Journal of the American Chemical Society 136, 13684 (2014).

[28] S. Bhattacharya, B. H. Sonin, C. J. Jumonville, L. M. Ghiringhelli, and N. Marom, Phys. Rev. B 91, 241115 (2015), URL https://link.aps.org/doi/10.1103/PhysRevB.91.241115

[29] N. Marom, M. Kim, and J. R. Chelikowsky, Phys. Rev. Lett. 108, 106801 (2012), URL https://link.aps.org/doi/10.1103/PhysRevLett.108.106801

[30] K. Fukui, T. Yonezawa, and H. Shingu, The Journal of Chemical Physics 20, 722 (1952), https://doi.org/10.1063/1.1700523, URL https://doi.org/10.1063/1.1700523.

[31] E. V. Anslyn and D. A. Dougherty, Modern physical organic chemistry (University science books, 2006).

[32] T. S. Khan, S. Gupta, M. I. Alam, and M. A. Haider, RSC Advances 6, 101697 (2016).

[33] O. Lopez-Acevedo, K. A. Kacprzak, J. Akola, and H. Häkkinen, Nature chemistry 2, 329 (2010).

[34] Z. S. Fishman, B. Rudshteyn, Y. He, B. Liu, S. Chaudhuri, M. Askerka, G. L. Haller, V. S. Batista, and L. D. Pfefferle, Journal of the American Chemical Society 138, 10978 (2016).

[35] P. Raybaud, J. Hafner, G. Kresse, and H. Toulhoat, Journal of Physics: Condensed Matter 9, 11107 (1997).

[36] J. A. Rodriguez, T. Jirsak, and S. Chaturvedi, The Journal of chemical physics 111, 8077 (1999).

[37] V. Blum, R. Gehrke, F. Hanke, P. Havu, V. Havu, X. Ren, K. Reuter, and M. Scheffler, Computer Physics Communications 180, 2175 (2009).

[38] A. Tkatchenko and M. Scheffler, Physical review letters 102, 073005 (2009).

[39] J. P. Perdew, M. Ernzerhof, and K. Burke, The Journal of Chemical Physics 105, 9982 (1996).

[40] M. Scheffler and U. Scherz, in Materials Science Forum (Trans Tech Publ, 1986), vol. 10, pp. 353-358.

[41] M. Scheffler and J. Dabrowski, Philosophical Magazine A 58, 107 (1988).

[42] X.-G. Wang, W. Weiss, S. K. Shaikhutdinov, M. Ritter, M. Petersen, F. Wagner, R. Schlögl, and M. Scheffler, Physical Review Letters 81, 1038 (1998).

[43] S.-H. Lee, W. Moritz, and M. Scheffler, Physical review letters 85, 3890 (2000).

[44] K. Reuter and M. Scheffler, Physical Review B 68, 045407 (2003).

[45] K. Reuter, C. Stampf, and M. Scheffler, in Handbook of Materials Modeling (Springer, 2005), pp. 149-194.

[46] S. Bhattacharya, D. Berger, K. Reuter, L. M. Ghiringhelli, and S. V. Levchenko, Phys. Rev. Materials 1, 071601 (2017), URL 
https://link.aps.org/doi/10.1103/PhysRevMaterials.1.07160ll $0.5 \mathrm{eV}$ from the global minimum as we have seen that

[47] A. Bhattacharya and S. Bhattacharya, The Journal of Physical Chemistry Letters 6, 3726 (2015), pMID: 26722747, https://doi.org/10.1021/acs.jpclett.5b01435, URL https://doi.org/10.1021/acs.jpclett.5b01435

[48] We varied $z$ value starting from $1,2,3, \ldots$ and kept on increasing it until the specific $\mathrm{TM}_{x} \mathrm{Mg}_{y} \mathrm{O}_{z}$ stoichiometry goes totally outside our phase diagrams, i.e. under no circumstances that stoichiometry can be important at realistic conditions

[49] As described in details in Ref [6, 28], in our cascade GA, the latter energy is used to evaluate the fitness function, i.e., a mapping of the energy interval between highestand lowest-energy cluster in the running pool into the interval $[0,1]$. Obviously, the higher the value of the fitness function for a cluster, the higher is the probability of selecting it for generating a new structure.

[50] We considered all the isomers within an energy window it's very unlikely that isomers above $0.5 \mathrm{eV}$ from the GM would become more stable after including their translational, rotational, vibrational, spin and symmetry free energy contributions to the total energy.

[51] The clusters are at their ground state configuration w. r. t. geometry and spin respectively.

[52] This phase diagram is plotted at the PBE+vdW level in order to show that the substrate plays crucial role in stability of configurations. The effect of advanced and accurate xc-functionals are introduced latter, while concluding our main results

[53] The energy window of $0.5 \mathrm{eV}$ in total energy is carefully chosen such that within this error bar in energetics, the phase diagram will not change significantly in terms of stability of different phases. 